\title{
Identifying and Addressing Barriers to Transgender Healthcare: Where We Are and What We Need to Do About It
}

\author{
David Michael Warner II, MS ${ }^{1,3}$ and Arunab Harish Mehta, MD 2,4 \\ 'Department of Physiology and Biophysics, Case Western Reserve University, Cleveland, OH, USA; ${ }^{2}$ Department of Hospital Medicine, Cleveland \\ Clinic Foundation, Cleveland, OH, USA; ${ }^{3}$ Beachwood, USA; ${ }^{4}$ Cleveland, USA.
}

KEY WORDS: transgender health; transgender healthcare; transgender health disparities; barriers to transgender healthcare; transgender healthcare barriers.

J Gen Intern Med 36(11):3559-61

DOI: $10.1007 / \mathrm{s} 11606-021-07001-2$

(c) Society of General Internal Medicine 2021

\section{INTRODUCTION}

The 2015 United States Transgender Survey (USTS) provided extensive data on the experiences of 27,715 adults with a variety of identities on the transgender spectrum, including transgender, trans, genderqueer, and non-binary. Among the conclusions provided, $33 \%$ of respondents seen by a healthcare provider within the year prior to completing the survey reported having at least one negative experience related to their gender identity. ${ }^{1}$

Approximately 1.4 million adults in the USA identify as transgender according to the Williams Institute. Compared to the general population, transgender and non-binary (TNB) individuals face higher rates of unemployment, poverty, homelessness, discrimination, psychological distress, substance abuse, HIV infection, suicide, and violence. ${ }^{2}$ The Human Rights Campaign reported that at least 44 TNB individuals were murdered in 2020, a majority of whom were black or Latinx. The medical community must come together to identify measures to address this public health crisis.

This viewpoint article will focus on the structural, interpersonal, and anticipation barriers to healthcare for TNB individuals and propose solutions to addressing these barriers.

\section{STRUCTURAL BARRIERS}

Structural barriers to transgender healthcare include high uninsured rates or lack of coverage for transgender-related services, inadequate training of physicians on transgendersensitive care, and limited access to providers who offer transgender-related care. ${ }^{2}$

Received January 14, 2021

Accepted June 24, 2021

Published online July 12, 2021
Between 1968 and 2013, gender identity disorder was listed in the Diagnostic and Statistical Manual of Mental Disorders. Treatments in the early 1900s focused on helping TNB individuals conform to binary gender expression rather than ameliorating the state of gender dysphoria experienced by some TNB individuals. ${ }^{2}$ Gender dysphoria is defined as distress caused by incongruence between sex assigned at birth and gender identity and is a formal diagnosis that is often required for healthcare reimbursement. ${ }^{2}$ This can create barriers to coverage if insurance providers deem gender-affirming interventions to be cosmetic or medically unnecessary or consider being transgender a preexisting condition. ${ }^{3}$ The 2015 USTS revealed that for those who sought transgender-related services, $25 \%$ of respondents were denied coverage for hormone therapy in the year prior, and 55\% were denied coverage for surgery in the year prior. ${ }^{1}$ In addition, $14 \%$ of respondents were uninsured, including $20 \%$ of black respondents. ${ }^{1}$ Lack of insurance coverage can prompt TNB individuals to resort to unregulated hormone use or silicone "pumping" to cope with gender dysphoria, which can cause serious complications. ${ }^{2}$

Inadequate coverage of transgender-related healthcare in medical education also provides a barrier to care for TNB individuals. The 2015 USTS showed that $24 \%$ of respondents had to teach their healthcare provider about transgender individuals to receive appropriate care. ${ }^{1}$ A 2011 study including the deans from 176 allopathic and osteopathic medical schools revealed that programs included in the study spent a median of $5 \mathrm{~h}$ and a mean of $7 \mathrm{~h}$ on LGBT-related health. ${ }^{4}$ In total, 33\% of schools included in the study required zero clinical hours for LGBT-related content. ${ }^{4}$ The sparse coverage of LGBT content in medical school curricula can impact physician knowledge, comfort, and attitudes when it comes to providing transgender care. One consequence is a limited number of providers who can provide adequate transgender-related care. The 2015 USTS revealed that respondents were three times more likely to have to travel 50 miles or more for transgenderrelated care than routine care. ${ }^{1}$

\section{INTERPERSONAL BARRIERS}

Interpersonal barriers to healthcare include lack of provider sensitivity or knowledge when providing transgender 
healthcare, refusal to care for transgender patients, and harassment.

Provider attitudes, sensitivity, and knowledge play an important role in the healthcare experiences of TNB individuals. In the 2015 USTS, verbal harassment, refusal of treatment, and having to teach healthcare providers about transgender health to receive appropriate care were cited as examples of negative experiences that $33 \%$ of respondents experienced within the year prior. ${ }^{1}$ A 2017 qualitative report on the healthcare provided to transgender and gender nonconforming (TGGNC) patients in emergency departments found that $45.7 \%$ of patients witnessed medical personnel gossiping, mocking, or telling jokes about TGGNC patients, $8.6 \%$ reported being outed by a healthcare professional, and $62.9 \%$ had an experience with healthcare staff refusing to use their correct pronouns. ${ }^{5}$ Negative experiences with healthcare providers can lead to mistrust in the medical system.

\section{ANTICIPATION BARRIERS}

Anticipation barriers include the avoidance of healthcare or health-promoting activities due to the anticipation of discrimination or mistreatment and stem from the structural and interpersonal barriers previously mentioned. The 2011 Report of the National Transgender Discrimination Survey (RNTDS) included 6450 TNB study participants. The 2011 RNTDS revealed that $28 \%$ of respondents postponed or avoided medical treatment when they were sick or injured due to fear of being discriminated against or disrespected by health providers. ${ }^{6}$ In addition, $33 \%$ of respondents postponed or avoided accessing preventive healthcare, which can cause adverse long-term health outcomes. ${ }^{6}$

Another anticipation barrier stemming from structural and interpersonal barriers is the avoidance of public restrooms. The 2015 USTS revealed that $59 \%$ of respondents reported avoiding public restrooms within the year prior. A total of $32 \%$ of respondents reported avoiding drinking or eating to avoid the need to use the restroom. Denial of access to restrooms and experiences with verbal, physical, and sexual assault in restrooms are among the structural and interpersonal barriers that contribute to the avoidance of public restrooms by TNB individuals. ${ }^{1}$

\section{RECOMMENDATIONS}

Steps that can be taken to address the structural, interpersonal, and anticipation barriers to transgender healthcare include enacting policy changes related to insurance coverage for gender-affirming healthcare, reforming medical school curricula to prepare future physicians to provide competent care to TNB patients, and continuing education for healthcare providers.

Currently, coverage decisions for gender-affirming medical care under Medicaid, Medicare, and many private insurances are dictated by states, which leads to inconsistency in coverage and constitutes an additional barrier to transgender healthcare. ${ }^{3}$ To address this inconsistency, healthcare providers and community members should educate themselves on their state and local laws and advocate for changes to improve insurance coverage for gender-affirming medical care.

Reforms to medical school curricula to prepare future physicians to provide competent care to TNB patients include increasing the number of hours devoted to the healthcare needs of TNB individuals and improving the quality of clinical experiences in medical school curricula. Additionally, conducting further research on best practices in transgenderrelated care would be helpful to identify and promote lasting structural changes.

Finally, healthcare professionals have a responsibility to increase their knowledge of transgender-related care and take measures to improve their sensitivity and professionalism when providing care to TNB patients. Creating a welcoming environment and cultivating patient trust by increasing provider sensitivity and knowledge regarding transgender health will help mitigate these individual barriers. Specific actions that can be taken by healthcare professionals include using correct names and pronouns, updating medical forms to include a greater diversity of sex and gender identities, avoiding gendered language, increasing awareness of the stressors experienced by TNB individuals, and ensuring that discussions on anatomy are medically relevant. Most importantly, healthcare providers should be open to continual learning, reflection, and growth when working with TNB patients to continue improving and providing optimal care.

\section{CONCLUSION}

There are existing inequities in the care provided to TNB individuals in our health system that contribute to adverse health outcomes. By identifying the structural, interpersonal, and anticipation barriers to transgender healthcare, we can make efforts to begin addressing the disparities in transgender care and promote health equity.

Corresponding Author: David Michael Warner, II, MS; Beachwood, USA (e-mail:dmw182@case.edu).

\section{Declarations:}

Conflict of Interest: The authors declare that they do not have a conflict of interest.

\section{REFERENCES}

1. James, SE, Herman, JL, Rankin, S, Keisling, M, Mottet, L, \& Anafi, M. (2016). The Report of the 2015 U.S. Transgender Survey. Washington, DC: National Center for Transgender Equality. 
2. White Hughto, JM, Reisner, SL, Pachankis, JE. Transgender Stigma and Health: A Critical Review of Stigma Determinants, Mechanisms, and Interventions. Soc Sci Med. 2015; 147: 222-231.

3. Bakko, M, Kattari, SK. Transgender-Related Insurance Denials as Barriers to Transgender Healthcare: Differences in Experience by Insurance Type. J Gen Intern Med. 2020; 35: 1693-1700.

4. Obedin-Maliver, J, Goldsmith, ES, Stewart, L, et al. Lesbian, Gay, Bisexual, and Transgender-Related Content in Undergraduate Medical Education. JAMA. 2011;306(9):971-977.

5. Chisolm-Straker, M, Jardine, L, Bennouna, C, et al. Transgender and Gender Nonconforming in Emergency Departments: A Qualitative Report of Patient Experiences. Transgender Health. 2017;2(1):8-16.
6. Grant, JM, Mottet, LA, Tanis, J, Harrison, J, Herman, JL, Keisling, M. (2011). Injustice at Every Turn: A Report of the National Transgender Discrimination Survey. Washington, DC: National Center for Transgender Equality and National Gay and Lesbian Task Force.

Publisher's Note Springer Nature remains neutral with regard to jurisdictional claims in published maps and institutional affiliations. 